\title{
Prime Filters and Ideals in Distributive Lattices
}

\author{
Adam Grabowski \\ Institute of Informatics \\ University of Białystok \\ Akademicka 2, 15-267 Białystok \\ Poland
}

Summary. The article continues the formalization of the lattice theory (as structures with two binary operations, not in terms of ordering relations). In the Mizar Mathematical Library, there are some attempts to formalize prime ideals and filters; one series of articles written as decoding [9] proven some results; we tried however to follow [21, [12, and [13. All three were devoted to the Stone representation theorem [18 for Boolean or Heyting lattices. The main aim of the present article was to bridge this gap between general distributive lattices and Boolean algebras, having in mind that the more general approach will eventually replace the common proof of aforementioned articles ${ }^{1}$

Because in Boolean algebras the notions of ultrafilters, prime filters and maximal filters coincide, we decided to construct some concrete examples of ultrafilters in nontrivial Boolean lattice. We proved also the Prime Ideal Theorem not as BPI (Boolean Prime Ideal), but in the more general setting.

In the final section we present Nachbin theorems [15, 1] expressed both in terms of maximal and prime filters and as the unordered spectra of a lattice 11, [10. This shows that if the notion of maximal and prime filters coincide in the lattice, it is Boolean.

MSC: 06D05 03B35

Keywords: prime filters; prime ideals; distributive lattices

MML identifier: LATTICEA, version: 8.1.02 5.19.1189

The notation and terminology used in this paper have been introduced in the following articles: [2], [3], [5], 6], 44, [24], 12], [7], [17], 22], 23], [16], 20], and [8].

\footnotetext{
${ }^{1}$ As one of the anonymous reviewers pointed out, it would be interesting to show counterexamples showing that the assumptions of the distributivity and boundedness are necessary, and this will be our plan for future work as basic examples of non-distributive lattices are available as of now only as relational structures. 


\section{Preliminaries}

Let $X$ be a set. We say that $X$ is unordered if and only if

(Def. 1) Let us consider sets $p_{1}, p_{2}$. Suppose

(i) $p_{1}, p_{2} \in X$, and

(ii) $p_{1} \neq p_{2}$.

Then $p_{1}$ and $p_{2}$ are $\subseteq$-incomparable.

Let us note that there exists a Boolean lattice which is non trivial.

Now we state the propositions:

(1) Let us consider a non trivial bounded lattice $L$. Then $\top_{L} \neq \perp_{L}$.

(2) Let us consider a lattice $L$ and an ideal $I$ of $L$. Then $I$ is prime if and only if $I^{\mathrm{c}}$ is a filter of $L$ or $I^{\mathrm{c}}=\emptyset$. Proof: If $I$ is prime, then $I^{\mathrm{c}}$ is a filter of $L$ or $I^{\mathrm{c}}=\emptyset$ by [20, (29)]. For every elements $x, y$ of $L, x \sqcap y \in I$ iff $x \in I$ or $y \in I$ by [2, (9), (8)].

(3) Let us consider a lattice $L$ and a filter $F$ of $L$. Then $F$ is prime if and only if $F^{\mathrm{c}}$ is an ideal of $L$ or $F^{\mathrm{c}}=\emptyset$. Proof: Set $F=I^{\mathrm{c}}$. If $I$ is prime, then $F$ is an ideal of $L$ or $F=\emptyset$ by [20, (29)]. For every elements $x, y$ of $L, x \sqcup y \in I$ iff $x \in I$ or $y \in I$ by [3, (21), (86)].

Let $L$ be a lattice. The functor PFilters $L$ yielding a family of subsets of $L$ is defined by the term

(Def. 2) $\quad\{F$, where $F$ is a filter of $L: F$ is prime $\}$.

Observe that $(L]$ is prime.

Now we state the proposition:

(4) Let us consider a distributive lattice $L$.

Then PrimeFilters $(L) \subset$ PFilters $L$. Proof: PrimeFilters $(L) \subseteq$ PFilters $L$. $[L) \notin \operatorname{PrimeFilters}(L)$.

\section{Examples of Filters in Nontrivial Boolean Lattices}

Now we state the propositions:

(5) The carrier of the lattice of subsets of $\{\emptyset\}=\{\emptyset,\{\emptyset\}\}$.

(6) Let us consider a lattice $L$ and a subset $A$ of $L$. Suppose $L=$ the lattice of subsets of $\{\emptyset\}$. Then

(i) $A=\emptyset$, or

(ii) $A=\{\emptyset\}$, or

(iii) $A=\{\emptyset,\{\emptyset\}\}$, or

(iv) $A=\{\{\emptyset\}\}$.

Let us consider a lattice $L$ and a filter $A$ of $L$. Now we state the propositions: 
(7) Suppose $L=$ the lattice of subsets of $\{\emptyset\}$. Then

(i) $A=\emptyset$, or

(ii) $A=\{\emptyset,\{\emptyset\}\}$, or

(iii) $A=\{\{\emptyset\}\}$.

(8) If $L=$ the lattice of subsets of $\{\emptyset\}$, then $A=\left\{\top_{L}\right\}$ or $A=[L)$.

Now we state the propositions:

(9) Let us consider a non trivial Boolean lattice $L$ and a filter $A$ of $L$. Suppose

(i) $L=$ the lattice of subsets of $\{\emptyset\}$, and

(ii) $A=\left\{\top_{L}\right\}$.

Then $A$ is prime. The theorem is a consequence of (5) and (7). Proof: For every filter $H$ of $L$ such that $A \subseteq H$ and $H \neq$ the carrier of $L$ holds $A=H$ by [4, (4)].

(10) Let us consider a lattice $L$ and a filter $A$ of $L$. Suppose

(i) $L=$ the lattice of subsets of $\{\emptyset\}$, and

(ii) $A$ is an ultrafilter.

Then $A=\left\{\top_{L}\right\}$. The theorem is a consequence of (7). Proof: $\emptyset \notin A$ by [4, (3)], [21, (29)].

\section{On Prime and Maximal Filters and Ideals}

Now we state the proposition:

(11) Let us consider a lattice $L$ and an element $a$ of $L$. Then $\{F$, where $F$ is a filter of $L: F$ is prime and $a \in F\} \subseteq$ PFilters $L$.

Let $L$ be a lattice and $F$ be a filter of $L$. We say that $F$ is maximal if and only if

(Def. 3) (i) $F$ is proper, and

(ii) for every filter $G$ of $L$ such that $G$ is proper and $F \subseteq G$ holds $F=G$.

One can check that every filter of $L$ which is maximal is also proper.

Observe that every filter of $L$ which is maximal is also an ultrafilter and every filter of $L$ which is an ultrafilter is also maximal.

Let $I$ be an ideal of $L$. We say that $I$ is maximal if and only if

(Def. 4) (i) $I$ is proper, and

(ii) for every ideal $J$ of $L$ such that $J$ is proper and $I \subseteq J$ holds $I=J$.

Now we state the proposition:

(12) Let us consider a lattice $L$ and an ideal $I$ of $L$. Then $I$ is max-ideal if and only if $I$ is maximal. Proof: For every ideal $J$ of $L$ such that $I \subseteq J$ and $J \neq$ the carrier of $L$ holds $I=J$. 
Let $L$ be a lattice. Observe that every ideal of $L$ which is maximal is also max-ideal and every ideal of $L$ which is max-ideal is also maximal.

Let us observe that every ideal of $L$ which is maximal is also proper.

Now we state the propositions:

(13) Let us consider a lattice $L$ and a filter $F$ of $L$. Suppose $F$ is not prime. Then there exist elements $a, b$ of $L$ such that

(i) $a \sqcup b \in F$, and

(ii) $a \notin F$, and

(iii) $b \notin F$.

(14) Let us consider a lattice $L$ and an ideal $F$ of $L$. Suppose $F$ is not prime. Then there exist elements $a, b$ of $L$ such that

(i) $a \sqcap b \in F$, and

(ii) $a \notin F$, and

(iii) $b \notin F$.

(15) Let us consider a lattice $L$, a filter $F$ of $L$, an element $a$ of $L$, and a set $G$. Suppose

(i) $G=\{x$, where $x$ is an element of $L$ : there exists an element $u$ of $L$ such that $u \in F$ and $a \sqcap u \sqsubseteq x\}$, and

(ii) $a \in G$.

Then $G$ is a filter of $L$. Proof: $G \subseteq$ the carrier of $L$. Reconsider $G_{1}=G$ as a subset of $L . G_{1}$ is meet-closed by [2, (5), (8)]. $G_{1}$ is final by [24, (7)].

(16) Let us consider a lattice $L$, an ideal $F$ of $L$, an element $a$ of $L$, and a set $G$. Suppose

(i) $G=\{x$, where $x$ is an element of $L$ : there exists an element $u$ of $L$ such that $u \in F$ and $x \sqsubseteq a \sqcup u\}$, and

(ii) $a \in G$.

Then $G$ is an ideal of $L$. Proof: $G \subseteq$ the carrier of $L$. $G$ is join-closed by [2, (4)], [3, (86)]. $G$ is initial by [24, (7)].

(17) Let us consider a distributive lattice $L$ and a filter $F$ of $L$. If $F$ is maximal, then $F$ is prime. The theorem is a consequence of (13) and (15). Proof: Consider $a, b$ being elements of $L$ such that $a \sqcup b \in F$ and $a \notin F$ and $b \notin F$. Set $G=\{x$, where $x$ is an element of $L$ : there exists an element $u$ of $L$ such that $u \in F$ and $a \sqcap u \sqsubseteq x\} . b \notin G$ by [2, (10), (8)], [24, (11)]. $F \subseteq G$ by [24, (6)].

Let $L$ be a distributive lattice. One can verify that every filter of $L$ which is maximal is also prime.

Now we state the proposition: 
(18) Let us consider a distributive lattice $L$ and an ideal $F$ of $L$. If $F$ is maximal, then $F$ is prime. The theorem is a consequence of (14) and (16). Proof: Consider $a, b$ being elements of $L$ such that $a \sqcap b \in F$ and $a \notin F$ and $b \notin F$. Set $G=\{x$, where $x$ is an element of $L$ : there exists an element $u$ of $L$ such that $u \in F$ and $x \sqsubseteq a \sqcup u\}$. $G \subseteq$ the carrier of $L$. $b \notin G$ by [3, (22), (21)], [24, (4)]. $F \subseteq G$ by [24, (5)].

Let $L$ be a distributive lattice. Observe that every ideal of $L$ which is maximal is also prime.

\section{Prime Ideal Theorem for Distributive Lattices}

Now we state the propositions:

(19) Prime ideal theorem for Distributive lattices:

Let us consider a distributive lattice $L$, an ideal $I$ of $L$, and a filter $F$ of $L$. Suppose $I$ misses $F$. Then there exists an ideal $P$ of $L$ such that

(i) $P$ is prime, and

(ii) $I \subseteq P$, and

(iii) $P$ misses $F$.

The theorem is a consequence of (14). Proof: Set $X=\{i$, where $i$ is an ideal of $L: I \subseteq i$ and $i$ misses $F\}$. For every set $Z$ such that $Z \neq \emptyset$ and $Z \subseteq X$ and $Z$ is $\subseteq$-linear holds $\bigcup Z \in X$ by [19, (1)], [8, (74)], [3, (21)]. Consider $Y$ being a set such that $Y \in X$ and for every set $Z$ such that $Z \in X$ and $Z \neq Y$ holds $Y \nsubseteq Z$. Consider $i$ being an ideal of $L$ such that $Y=i$ and $I \subseteq i$ and $i$ misses $F$. $i$ is prime by [3, (50), (28)], [2, (1), (9), $(8)]$.

(20) Let us consider a distributive lattice $L$, an ideal $I$ of $L$, and an element $a$ of $L$. Suppose $a \notin I$. Then there exists an ideal $P$ of $L$ such that

(i) $P$ is prime, and

(ii) $I \subseteq P$, and

(iii) $a \notin P$.

The theorem is a consequence of (19). Proof: Set $F=[a)$. I misses $F$ by [2, (15)], [3, (21)]. Consider $P$ being an ideal of $L$ such that $P$ is prime and $I \subseteq P$ and $P$ misses $F$.

Let us consider a distributive lattice $L$ and elements $a, b$ of $L$. Now we state the propositions:

(21) If $a \neq b$, then there exists an ideal $P$ of $L$ such that $P$ is prime and $a \in P$ and $b \notin P$ or $a \notin P$ and $b \in P$.

(22) If $a \nsubseteq b$, then there exists an ideal $P$ of $L$ such that $P$ is prime and $a \notin P$ and $b \in P$. 
Now we state the proposition:

(23) Let us consider a distributive lattice $L$ and an ideal $I$ of $L$. Then $I=$ $\bigcap\{P$, where $P$ is an ideal of $L: P$ is prime and $I \subseteq P\}$. The theorem is a consequence of $(20)$. Proof: $\Omega_{L}$ is prime.

\section{The Stone Representation}

Let $L$ be a lattice. The prime filters of $L$ yielding a function is defined by

(Def. 5) (i) dom $i t=$ the carrier of $L$, and

(ii) for every element $a$ of $L, i t(a)=\{F$, where $F$ is a filter of $L$ : $F$ is prime and $a \in F\}$.

Now we state the propositions:

(24) Let us consider a lattice $L$, an element $a$ of $L$, and a set $x$. Then $x \in$ (the prime filters of $L)(a)$ if and only if there exists a filter $F$ of $L$ such that $F=x$ and $F$ is prime and $a \in F$. Proof: If $x \in$ (the prime filters of $L)(a)$, then there exists a filter $F$ of $L$ such that $F=x$ and $F$ is prime and $a \in F$.

(25) Let us consider a lattice $L$, an element $a$ of $L$, and a filter $F$ of $L$. Then $F \in($ the prime filters of $L)(a)$ if and only if $F$ is prime and $a \in F$. The theorem is a consequence of $(24)$.

Let us consider a distributive lattice $L$ and elements $a, b$ of $L$. Now we state the propositions:

(26) (The prime filters of $L)(a \sqcap b)=($ the prime filters of $L)(a) \cap$ (the prime filters of $L)(b)$.

(27) (The prime filters of $L)(a \sqcup b)=($ the prime filters of $L)(a) \cup$ (the prime filters of $L)(b)$.

Let $L$ be a distributive lattice. Let us note that the prime filters of $L$ yields a function from the carrier of $L$ into $2^{\text {PFilters } L}$. The functor $\operatorname{StoneR}(L)$ yielding a set is defined by the term

(Def. 6) rng the prime filters of $L$.

Note that $\operatorname{StoneR}(L)$ is non empty.

Now we state the proposition:

(28) Let us consider a distributive lattice $L$ and a set $x$. Then $x \in \operatorname{StoneR}(L)$ if and only if there exists an element $a$ of $L$ such that (the prime filters of $L)(a)=x$. Proof: If $x \in \operatorname{StoneR}(L)$, then there exists an element $a$ of $L$ such that (the prime filters of $L)(a)=x$.

Let $L$ be an upper-bounded distributive lattice. The functor $\operatorname{StoneSpace}(L)$ yielding a strict topological space is defined by

(Def. 7) (i) the carrier of $i t=$ PFilters $L$, and 
(ii) the topology of it $=$

$\{\bigcup A$, where $A$ is a family of subsets of PFilters $L: A \subseteq \operatorname{StoneR}(L)\}$.

Let $L$ be a non trivial upper-bounded distributive lattice. One can check that $\operatorname{StoneSpace}(L)$ is non empty.

\section{Pseudo Complements in Lattices}

Let $L$ be a lattice and $a$ be an element of $L$. The functors: the set of pseudocomplements of $a$ and the set of dual pseudo-complements of $a$ yielding subsets of $L$ are defined by terms, respectively.

(Def. 8) $\quad\left\{x\right.$, where $x$ is an element of $\left.L: a \sqcap x=\perp_{L}\right\}$.

(Def. 9) $\left\{x\right.$, where $x$ is an element of $\left.L: a \sqcup x=\top_{L}\right\}$.

Let $L$ be a distributive bounded lattice.

Note that the set of pseudo-complements of $a$ is initial non empty and join-closed and the set of dual pseudo-complements of $a$ is final non empty and meet-closed.

Let us consider a lattice $L$ and elements $a, b$ of $L$. Now we state the propositions:

(29) $b \in$ the set of pseudo-complements of $a$ if and only if $b \sqcap a=\perp_{L}$.

(30) $b \in$ the set of dual pseudo-complements of $a$ if and only if $b \sqcup a=\top_{L}$.

Let us consider a bounded lattice $L$ and an element $a$ of $L$. Now we state the propositions:

(31) $\perp_{L} \in$ the set of pseudo-complements of $a$.

(32) $\top_{L} \in$ the set of dual pseudo-complements of $a$.

\section{NAChBin's Theorem for Bounded Distributive Lattices}

Let $L$ be a lattice. The spectrum of $L$ yielding a family of subsets of $L$ is defined by the term

(Def. 10) $\{I$, where $I$ is an ideal of $L: I$ is prime and proper $\}$.

Now we state the proposition:

(33) NACHBIN'S THEOREM FOR BOUNDED DisTRIBUTIVE LATTICES:

Let us consider a distributive bounded lattice $L$. Then $L$ is Boolean if and only if for every ideal $I$ of $L$ such that $I$ is proper and prime holds $I$ is maximal. The theorem is a consequence of (19). Proof: If $L$ is Boolean, then for every ideal $I$ of $L$ such that $I$ is proper and prime holds $I$ is maximal by [3, (57)]. Consider $a$ being an element of $L$ such that there exists no an element $b$ of $L$ such that $b$ is a complement of $a$. Set $I_{0}=$ the set of pseudo-complements of $a$. Set $I_{1}=\{x$, where $x$ is an element of $L$ : there exists an element $y$ of $L$ such that $y \in I_{0}$ and $\left.x \sqsubseteq a \sqcup y\right\}$. 
$I_{1} \subseteq$ the carrier of $L$. For every elements $p, q$ of $L$ such that $p \sqsubseteq q$ and $q \in I_{1}$ holds $p \in I_{1}$ by [24, (7)]. For every elements $p, q$ of $L$ such that $p, q \in I_{1}$ holds $p \sqcup q \in I_{1}$ by [2, (4)]. $I_{0} \subseteq I_{1}$ by [24, (5)]. $\top_{L} \notin I_{1}$. Set $F_{2}=\left[\top_{L}\right)$. Consider $J_{0}$ being an ideal of $L$ such that $J_{0}$ is prime and $I_{1} \subseteq J_{0}$ and $J_{0}$ misses $F_{2}$. Set $T=$ the carrier of $L$. Reconsider $D=T \backslash J_{0}$ as a non empty subset of $L$. For every elements $p, q$ of $L$ such that $p \sqsubseteq q$ and $p \in D$ holds $q \in D$ by [3, (21)]. For every elements $p, q$ of $L$ such that $p, q \in D$ holds $p \sqcap q \in D$. Reconsider $F=[[a) \cup D)$ as a filter of $L$. $F$ misses $I_{0}$ by [13, (3)], [24, (6)], [14, (9)]. Consider $J_{1}$ being an ideal of $L$ such that $J_{1}$ is prime and $I_{0} \subseteq J_{1}$ and $J_{1}$ misses $F$. $J_{1} \subseteq J_{0}$.

Let $L$ be a non trivial distributive bounded lattice. Let us note that the spectrum of $L$ is non empty.

Now we state the proposition:

(34) NACHBin theOREM For SPECTRA OF Distributive LATtices:

Let us consider a distributive bounded lattice $L$. Then $L$ is Boolean if and only if the spectrum of $L$ is unordered. The theorem is a consequence of (19) and (20). Proof: If $L$ is Boolean, then the spectrum of $L$ is unordered by [3, (57), (58)], [24, (20)]. Consider $a$ being an element of $L$ such that there exists no an element $b$ of $L$ such that $b$ is a complement of $a$. Set $D=$ the set of dual pseudo-complements of $a$. Set $D_{1}=[D \cup[a)) . D_{1} \subseteq$ $\{x$, where $x$ is an element of $L$ : there exists an element $d$ of $L$ such that $d \in D$ and $a \sqcap d \sqsubseteq x\}$ by [2, (15), (5)], [24, (7)]. \{x, where $x$ is an element of $L$ : there exists an element $d$ of $L$ such that $d \in D$ and $a \sqcap d \sqsubseteq x\} \subseteq$ $D_{1} \cdot \perp_{L} \notin D_{1}$ by [24, (8)]. Reconsider $I_{0}=\left\{\perp_{L}\right\}$ as an ideal of $L$. Consider $P$ being an ideal of $L$ such that $P$ is prime and $I_{0} \subseteq P$ and $P$ misses $D_{1}$. Set $P_{1}=(P \cup(a]]$. $\top_{L} \notin P_{1}$ by [3, (49)], [2, (1)], [3, (28)]. Consider $Q$ being an ideal of $L$ such that $Q$ is prime and $P_{1} \subseteq Q$ and $\top_{L} \notin Q$.

Let $L$ be a Boolean lattice. Note that the spectrum of $L$ is unordered.

\section{REFERENCES}

[1] Raymond Balbes and Philip Dwinger. Distributive Lattices. University of Missouri Press, 1975.

[2] Grzegorz Bancerek. Filters - part I. Formalized Mathematics, 1(5):813-819, 1990.

[3] Grzegorz Bancerek. Ideals Formalized Mathematics, 5(2):149-156, 1996.

[4] Grzegorz Bancerek. Complete lattices. Formalized Mathematics, 2(5):719-725, 1991.

[5] Czesław Byliński. Functions and their basic properties Formalized Mathematics, 1(1): 55-65, 1990.

[6] Czesław Byliński. Functions from a set to a set Formalized Mathematics, 1(1):153-164, 1990.

[7] Czesław Byliński. Partial functions Formalized Mathematics, 1(2):357-367, 1990.

[8] Czesław Byliński. Some basic properties of sets Formalized Mathematics, 1(1):47-53, 1990.

[9] G. Gierz, K.H. Hofmann, K. Keimel, J.D. Lawson, M. Mislove, and D.S. Scott. A Compendium of Continuous Lattices. Springer-Verlag, Berlin, Heidelberg, New York, 1980. 
[10] George Grätzer. General Lattice Theory. Academic Press, New York, 1978.

[11] George Grätzer. Lattice Theory: Foundation. Birkhäuser, 2011.

[12] Jolanta Kamieńska. Representation theorem for Heyting lattices Formalized Mathematics, 4(1):41-45, 1993.

[13] Jolanta Kamieńska and Jarosław Stanisław Walijewski. Homomorphisms of lattices, finite join and finite meet Formalized Mathematics, 4(1):35-40, 1993.

[14] Agnieszka Julia Marasik. Boolean properties of lattices Formalized Mathematics, 5(1): 31-35, 1996.

[15] Leopoldo Nachbin. Une propriété characteristique des algebres booleiennes. Portugaliae Mathematica, 6:115-118, 1947.

[16] Beata Padlewska. Families of sets. Formalized Mathematics, 1(1):147-152, 1990.

[17] Beata Padlewska and Agata Darmochwał. Topological spaces and continuous functions Formalized Mathematics, 1(1):223-230, 1990.

[18] Marshall H. Stone. The theory of representations of Boolean algebras. Transactions of the American Mathematical Society, 40:37-111, 1936.

[19] Andrzej Trybulec. Tarski Grothendieck set theory Formalized Mathematics, 1(1):9-11, 1990.

[20] Zinaida Trybulec. Properties of subsets. Formalized Mathematics, 1(1):67-71, 1990.

[21] Jarosław Stanisław Walijewski. Representation theorem for Boolean algebras Formalized Mathematics, 4(1):45-50, 1993.

[22] Edmund Woronowicz. Relations and their basic properties Formalized Mathematics, 1 (1):73-83, 1990.

[23] Edmund Woronowicz. Relations defined on sets Formalized Mathematics, 1(1):181-186, 1990.

[24] Stanisław Żukowski. Introduction to lattice theory Formalized Mathematics, 1(1):215$222,1990$.

Received October 7, 2013 